\title{
AN INVARIANT SUBSPACE THEOREM AND INVARIANT SUBSPACES OF ANALYTIC REPRODUCING KERNEL HILBERT SPACES - I
}

\author{
JAYDEB SARKAR
}

\begin{abstract}
Let $T$ be a $C_{\cdot 0}$-contraction on a Hilbert space $\mathcal{H}$ and $\mathcal{S}$ be a non-trivial closed subspace of $\mathcal{H}$. We prove that $\mathcal{S}$ is a $T$-invariant subspace of $\mathcal{H}$ if and only if there exists a Hilbert space $\mathcal{D}$ and a partially isometric operator $\Pi: H_{\mathcal{D}}^{2}(\mathbb{D}) \rightarrow \mathcal{H}$ such that $\Pi M_{z}=T \Pi$ and that $\mathcal{S}=$ ran $\Pi$, or equivalently,

$$
P_{\mathcal{S}}=\Pi \Pi^{*} .
$$

As an application we completely classify the shift-invariant subspaces of analytic reproducing kernel Hilbert spaces over the unit disc. Our results also includes the case of weighted Bergman spaces over the unit disk.
\end{abstract}

\section{INTRODUCTION}

One of the most famous open problems in operator theory and function theory is the socalled invariant subspace problem: Let $T$ be a bounded linear operator on a Hilbert space $\mathcal{H}$. Does there exist a proper non-trivial closed subspace $\mathcal{S}$ of $\mathcal{H}$ such that $T \mathcal{S} \subseteq \mathcal{S}$ ?

A paradigm is the well-known fact, due to Beurling, Lax and Halmos (see [3], [9], [7] and [11]), that any shift-invariant subspace of $H_{\mathcal{E}}^{2}(\mathbb{D})$ is given by an isometric, or partially isometric, image of a vector-valued Hardy space. Moreover, the isometry, or the partial isometry, can be realized as an operator-valued bounded holomorphic function on $\mathbb{D}$. More precisely, let $\mathcal{S}$ be a non-trivial closed subspaces of $H_{\mathcal{E}}^{2}(\mathbb{D})$. Then $\mathcal{S}$ is shift-invariant if and only if there exists a Hilbert space $\mathcal{E}_{*}$ such that $\mathcal{S}$ is the range of an isometric, or partially isometric operator from $H_{\mathcal{E}_{*}}^{2}(\mathbb{D})$ to $H_{\mathcal{E}}^{2}(\mathbb{D})$ which intertwine the shift operators (see [13]). Here $\mathcal{E}$ is a separable Hilbert space and $H_{\mathcal{E}}^{2}(\mathbb{D})$ denote the $\mathcal{E}$-valued Hardy space over the unit disc $\mathbb{D}=\{z \in \mathbb{C}:|z|<1\}$ (see [1], [13]).

In this paper we extend the Beurling-Lax-Halmos theorem for shift invariant subspaces of vector-valued Hardy spaces to the context of invariant subspaces of arbitrary $C .0^{-}$-contractions. Recall that a contraction $T$ on $\mathcal{H}$ (that is, $\|T f\| \leq\|f\|$ for all $f \in \mathcal{H}$ ) is said to be a $C \cdot 0^{-}$ contraction if $T^{* m} \rightarrow 0$ as $m \rightarrow \infty$ in the strong operator topology. One of our main results, Theorem 2.2, state that: Let $\mathcal{S}$ be a non-trivial closed subspace of a Hilbert space $\mathcal{H}$ and

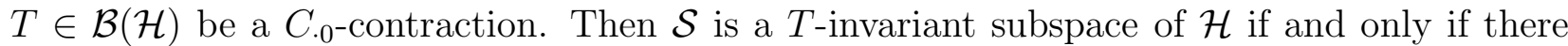
exists a Hilbert space $\mathcal{E}$ and a partial isometry $\Pi: H_{\mathcal{E}}^{2}(\mathbb{D}) \rightarrow \mathcal{H}$ such that $\Pi M_{z}=T \Pi$ and that $\mathcal{S}=\Pi H_{\mathcal{E}}^{2}(\mathbb{D})$. This theorem will be proven in Section 2 .

2010 Mathematics Subject Classification. 30H05, 46E22, 46M05, 46N99, 47A20, 47A45, 47B32, $47 \mathrm{~B} 38$.

Key words and phrases. Reproducing kernels, Hilbert modules, invariant subspaces, weighted Bergman spaces, Hardy space. 
In Section 3 we specialize to the case of reproducing kernel Hilbert spaces, in which $T=$ $M_{z} \otimes I_{\mathcal{E}_{*}}$ and $\mathcal{H}=\mathcal{H}_{K} \otimes \mathcal{E}_{*}$. Here $\mathcal{H}_{K}$ is an analytic Hilbert space (see Definition 3.11) and $\mathcal{E}_{*}$ is a coefficient space. In Theorem 3.3 we show that a non-trivial closed subspace $\mathcal{S}$ of $\mathcal{H}_{K} \otimes \mathcal{E}_{*}$ is $M_{z} \otimes I_{\mathcal{E}_{*}}$-invariant if and only if there exists a Hilbert space $\mathcal{E}$ and a partially isometric multiplier $\Theta \in \mathcal{M}\left(H^{2}(\mathbb{D}) \otimes \mathcal{E}, \mathcal{H}_{K} \otimes \mathcal{E}_{*}\right)$ such that

$$
\mathcal{S}=\Theta H_{\mathcal{E}}^{2}(\mathbb{D})
$$

This classification extends the results of Olofsson, Ball and Bolotnikov in [12, [4] and [5] on the shift-invariant subspaces of vector-valued weighted Bergman spaces with integer weights to that of vector-valued analytic Hilbert spaces.

Our approach has two main ingredients: the Sz.-Nagy and Foias dilation theory [11] and Hilbert module approach to operator theory [6]. However, to avoid technical complications we speak here just of operators on Hilbert spaces instead of Hilbert modules over function algebras.

Finally, it is worth mentioning that in the study of invariant subspaces of bounded linear operators on Hilbert spaces we lose no generality if we restrict our attention to the class of C.0-contractions.

Notations: (1) All Hilbert spaces considered in this paper are separable and over $\mathbb{C}$. We denote the set of natural numbers including zero by $\mathbb{N}$. (2) Let $\mathcal{H}$ be a Hilbert space and $\mathcal{S}$ be a closed subspace of $\mathcal{H}$. The orthogonal projection of $\mathcal{H}$ onto $\mathcal{S}$ is denoted by $P_{\mathcal{S}}$. (3) Let $\mathcal{H}_{1}, \mathcal{H}_{2}$ and $\mathcal{H}$ be Hilbert spaces. We denote by $\mathcal{B}\left(\mathcal{H}_{1}, \mathcal{H}_{2}\right)$ the set of all bounded linear operators from $\mathcal{H}_{1}$ to $\mathcal{H}_{2}$ and $\mathcal{B}(\mathcal{H})=\mathcal{B}(\mathcal{H}, \mathcal{H})$. (4) Two operators $T_{1} \in \mathcal{B}\left(\mathcal{H}_{1}\right)$ and $T_{2} \in \mathcal{B}\left(\mathcal{H}_{2}\right)$ are said to be unitarily equivalent, denoted by $T_{1} \cong T_{2}$, if there exists a unitary operator $U \in \mathcal{B}\left(\mathcal{H}_{1}, \mathcal{H}_{2}\right)$ such that $U T_{1}=T_{2} U$. (5) Let $\mathcal{E}$ be a Hilbert space. We will often identify $H_{\mathcal{E}}^{2}(\mathbb{D})$ with $H^{2}(\mathbb{D}) \otimes \mathcal{E}$ and $M_{z} \in \mathcal{B}\left(H_{\mathcal{E}}^{2}(\mathbb{D})\right)$ with $M_{z} \otimes I_{\mathcal{E}} \in \mathcal{B}\left(H^{2}(\mathbb{D}) \otimes \mathcal{E}\right)$, via the unitary $U \in \mathcal{B}\left(H_{\mathcal{E}}^{2}(\mathbb{D}), H^{2}(\mathbb{D}) \otimes \mathcal{E}\right.$ ) where $U\left(z^{m} \eta\right)=z^{m} \otimes \eta$ and $m \in \mathbb{N}$ and $\eta \in \mathcal{E}$.

\section{An InVARIAnt Subspace THEOREM}

In this section we will present a generalization of the Beurling-Lax-Halmos theorem to the class of $C \cdot 0^{-}$-contractions on Hilbert spaces.

Let $T$ be a $C_{.0}$-contraction on a Hilbert space $\mathcal{H}$. A fundamental theorem of Sz.-Nagy and Foias says that

$$
\left.T \cong P_{\mathcal{Q}} M_{z}\right|_{\mathcal{Q}}
$$

where $\mathcal{Q}$ is a $M_{z}^{*}$-invariant subspace of $H_{\mathcal{D}}^{2}(\mathbb{D})$ for some coefficient Hilbert space $\mathcal{D}$. In the following, we state and prove a variant of this fact which is adapted to our present purposes.

THEOREM 2.1. Let $T$ be a $C_{\cdot 0}$-contraction on a Hilbert space $\mathcal{H}$. Then there exists a coefficient Hilbert space $\mathcal{D}$ and a co-isometry $\Pi_{T}: H_{\mathcal{D}}^{2}(\mathbb{D}) \rightarrow \mathcal{H}$ such that $T \Pi_{T}=\Pi_{T} M_{z}$.

Proof. Let $D=\left(I_{\mathcal{H}}-T T^{*}\right)^{\frac{1}{2}}$ and $\mathcal{D}=\overline{\operatorname{ran}} D$. Since $\left\|z T^{*}\right\|_{\mathcal{B}(\mathcal{H})}=|z|\left\|T^{*}\right\|_{\mathcal{B}(\mathcal{H})}<1$, the inverse of $I_{\mathcal{H}}-z T^{*}$ exists in $\mathcal{B}(\mathcal{H})$ for all $z \in \mathbb{D}$. 
Define $L_{T}: \mathcal{H} \rightarrow H_{\mathcal{D}}^{2}(\mathbb{D})$ by

$$
\left(L_{T} h\right)(z):=D\left(I_{\mathcal{H}}-z T^{*}\right)^{-1} h=\sum_{m=0}^{\infty}\left(D T^{* m} h\right) z^{m} . \quad(h \in \mathcal{H}, z \in \mathbb{D})
$$

Now we compute

$$
\begin{aligned}
\left\|L_{T} h\right\|^{2} & =\left\|\sum_{m=0}^{\infty}\left(D T^{* m} h\right) z^{m}\right\|^{2}=\sum_{m=0}^{\infty}\left\|D T^{* m} h\right\|^{2}=\sum_{m=0}^{\infty}\left\langle T^{m} D^{2} T^{* m} h, h\right\rangle \\
& =\sum_{m=0}^{\infty}\left\langle T^{m}\left(I_{\mathcal{H}}-T T^{*}\right) T^{* m} h, h\right\rangle=\sum_{m=0}^{\infty}\left(\left\|T^{* m} h\right\|^{2}-\left\|T^{*(m+1)} h\right\|^{2}\right) \\
& =\|h\|^{2}-\lim _{m \rightarrow \infty}\left\|T^{* m} h\right\|^{2},
\end{aligned}
$$

where the last equality follows from the fact that the sum is a telescoping series. This and the fact that $\lim _{m \rightarrow \infty} T^{* m}=0$, in the strong operator topology, implies that

$$
\left\|L_{T} h\right\|=\|h\| . \quad(h \in \mathcal{H})
$$

Thus $L_{T}$ is an isometry and $\Pi_{T}: H_{\mathcal{D}}^{2}(\mathbb{D}) \rightarrow \mathcal{H}$ defined by $\Pi_{T}=L_{T}^{*}$ is a co-isometry. Finally, for all $h \in \mathcal{H}, \eta \in \mathcal{D}$ and $m \in \mathbb{N}$ we have

$$
\begin{aligned}
\left\langle\Pi_{T}\left(z^{m} \eta\right), h\right\rangle_{\mathcal{H}} & =\left\langle z^{m} \eta, D\left(I_{\mathcal{H}}-z T^{*}\right)^{-1} h\right\rangle_{H_{\mathcal{D}}^{2}(\mathbb{D})}=\left\langle z^{m} \eta, \sum_{l \in \mathbb{N}}\left(D T^{* l} h\right) z^{l}\right\rangle_{H_{\mathcal{D}}^{2}(\mathbb{D})} \\
& =\left\langle\eta, D T^{* m} h\right\rangle_{\mathcal{H}}=\left\langle T^{m} D \eta, h\right\rangle_{\mathcal{H}},
\end{aligned}
$$

that is,

$$
\Pi_{T}\left(z^{m} \eta\right)=T^{m} D \eta
$$

Therefore, for each $m \in \mathbb{N}$ and $\eta \in \mathcal{D}$ we have

$$
\Pi_{T} M_{z}\left(z^{m} \eta\right)=\Pi_{T}\left(z^{m+1} \eta\right)=T^{m+1} D \eta=T\left(T^{m} D \eta\right)=T \Pi_{T}\left(z^{m} \eta\right) .
$$

Since $\left\{z^{m} \eta: m \in \mathbb{N}, \eta \in \mathcal{D}\right\}$ is total in $H_{\mathcal{D}}^{2}(\mathbb{D})$, it follows that

$$
\Pi_{T} M_{z}=T \Pi_{T}
$$

The proof is now complete.

Now we present the main theorem of this section.

TheOREM 2.2. Let $T \in \mathcal{B}(\mathcal{H})$ be a $C_{.0}$-contraction and $\mathcal{S}$ be a non-trivial closed subspace of $\mathcal{H}$. Then $\mathcal{S}$ is a $T$-invariant subspace of $\mathcal{H}$ if and only if there exists a Hilbert space $\mathcal{D}$ and a partially isometric operator $\Pi: H_{\mathcal{D}}^{2}(\mathbb{D}) \rightarrow \mathcal{H}$ such that

$$
\Pi M_{z}=T \Pi,
$$

and that

$$
\mathcal{S}=\operatorname{ran} \Pi
$$


Proof. If $\mathcal{S}$ is a $T$-invariant subspace of $\mathcal{H}$ then

$$
\left(\left.T\right|_{\mathcal{S}}\right)^{* m}=\left.P_{\mathcal{S}} T^{* m}\right|_{\mathcal{S}}=P_{\mathcal{S}} T^{* m}
$$

shows that

$$
\left\|\left(\left.T\right|_{\mathcal{S}}\right)^{* m} f\right\|=\left\|P_{\mathcal{S}} T^{* m} f\right\| \leq\left\|T^{* m} f\right\|,
$$

for all $f \in \mathcal{S}$ and $m \in \mathbb{N}$. Thus $\left.T\right|_{\mathcal{S}} \in \mathcal{B}(\mathcal{S})$ is a $C_{\text {. }}$-contraction. Now Theorem 2.1 implies that there exists a Hilbert space $\mathcal{D}$ and a co-isometric map

$$
\Pi_{\left.T\right|_{\mathcal{S}}}: H_{\mathcal{D}}^{2}(\mathbb{D}) \rightarrow \mathcal{S}
$$

such that

$$
\Pi_{\left.T\right|_{\mathcal{S}}} M_{z}=\left.T\right|_{\mathcal{S}} \Pi_{\left.T\right|_{\mathcal{S}}} .
$$

Obviously the inclusion map $i: \mathcal{S} \rightarrow \mathcal{H}$ is an isometry and

$$
\left.i T\right|_{\mathcal{S}}=T i .
$$

Let $\Pi$ be the bounded linear map from $H_{\mathcal{D}}^{2}(\mathbb{D})$ to $\mathcal{H}$ defined by $\Pi=i \Pi_{\left.T\right|_{\mathcal{S}}}$. Then

$$
\Pi \Pi^{*}=\left(i \Pi_{\left.T\right|_{\mathcal{S}}}\right)\left(\Pi_{\left.T\right|_{\mathcal{S}}}^{*} i^{*}\right)=i i^{*}=P_{\mathcal{S}} .
$$

Therefore $\Pi$ is a partial isometry and $\operatorname{ran} \Pi=\mathcal{S}$. Finally,

$$
\Pi M_{z}=i \Pi_{\left.T\right|_{\mathcal{S}}} M_{z}=\left.i T\right|_{\mathcal{S}} \Pi_{\left.T\right|_{\mathcal{S}}}=T i \Pi_{\left.T\right|_{\mathcal{S}}}=T \Pi \text {. }
$$

This proves the necessary part.

To prove the sufficient part it is enough to note that ranП is a closed subspace of $\mathcal{H}$ and $T \Pi=\Pi M_{z}$ implies that ran $\Pi$ is a $T$-invariant subspace of $\mathcal{H}$. This completes the proof.

The following corollary is a useful variation of the invariant subspace theorem:

Corollary 2.3. Let $T \in \mathcal{B}(\mathcal{H})$ be a $C_{.0}$-contraction and $\mathcal{S}$ be a non-trivial closed subspace of $\mathcal{H}$. Then $\mathcal{S}$ is a $T$-invariant subspace of $\mathcal{H}$ if and only if there exists a Hilbert space $\mathcal{D}$ and a bounded linear operator $\Pi: H_{\mathcal{D}}^{2}(\mathbb{D}) \rightarrow \mathcal{H}$ such that $\Pi M_{z}=T \Pi$ and

$$
P_{\mathcal{S}}=\Pi \Pi^{*} \text {. }
$$

Before we go into the general theory of invariant subspaces of reproducing kernel Hilbert spaces, let us consider the classical Beurling-Lax-Halmos theorem as a simple corollary of Theorem 2.2.

Corollary 2.4. Let $\mathcal{S}$ be a non-trivial closed subspace of the Hardy space $H_{\mathcal{E}}^{2}(\mathbb{D})$. Then $\mathcal{S}$ is $M_{z}$-invariant if and only if there exists a Hilbert space $\mathcal{F}$ and a multiplier $\Theta \in H_{\mathcal{L}(\mathcal{F}, \mathcal{E})}^{\infty}(\mathbb{D})$ such that $M_{\Theta}: H_{\mathcal{F}}^{2}(\mathbb{D}) \rightarrow H_{\mathcal{E}}^{2}(\mathbb{D})$ is partially isometric and $\mathcal{S}=\Theta H_{\mathcal{F}}^{2}(\mathbb{D})$.

Proof. Let $\mathcal{F}$ be a Hilbert space and $X: H_{\mathcal{F}}^{2}(\mathbb{D}) \rightarrow H_{\mathcal{E}}^{2}(\mathbb{D})$ be a bounded linear map. It is easy to see that $X\left(M_{z} \otimes I_{\mathcal{F}}\right)=\left(M_{z} \otimes I_{\mathcal{E}}\right) X$ if and only if $X=M_{\Theta}$, the multiplication operator by $\Theta$, for some multiplier $\Theta \in H_{\mathcal{L}(\mathcal{F}, \mathcal{E})}^{\infty}(\mathbb{D})$. Now the result follows directly from Theorem 2.2 ,

Finally, note that Theorem 2.2 can be viewed as a generalization of the Beurling-LaxHalmos theorem for shift-invariant subspaces of vector-valued Hardy spaces to invariant subspaces of $C_{\cdot 0}$-contractions on Hilbert spaces. 


\section{Analytic Hilbert spaces}

Let $\mathcal{H}$ be a reproducing kernel Hilbert space of $\mathcal{E}$-valued holomorphic functions on $\mathbb{D}$ such that the multiplication operator by the coordinate function, denoted by $M_{z}$, is bounded on $\mathcal{H}$ (cf. [2]). A closed subspace $\mathcal{S}$ of $\mathcal{H}$ is said to be shift-invariant provided the product $z f \in \mathcal{S}$ whenever $f \in \mathcal{S}$.

The most general result on shift invariant subspaces has recently been obtained by Olofsson, Ball and Bolotnikov in [12], [4] and [5]. Namely, for a given Hilbert space $\mathcal{E}_{*}$, a closed subspace $\mathcal{S}$ of the weighted Bergman space $L_{a, m}^{2}(\mathbb{D}) \otimes \mathcal{E}_{*}(m \geq 2$ and $m \in \mathbb{N})$ is shift-invariant if and only if there exists a Hilbert space $\mathcal{E}$ and a function $\Theta: \mathbb{D} \rightarrow \mathcal{B}\left(\mathcal{E}, \mathcal{E}_{*}\right)$ such that $M_{\Theta}: H^{2}(\mathbb{D}) \otimes \mathcal{E} \rightarrow L_{a, m}^{2}(\mathbb{D}) \otimes \mathcal{E}_{*}$ is a multiplier (see definition below) and $\mathcal{S}=\Theta H_{\mathcal{E}}^{2}(\mathbb{D})$. Recall that the weighted Bergman space $L_{a, \alpha}^{2}(\mathbb{D})$, with $\alpha>1$, is a reproducing kernel Hilbert space corresponding to the kernel

$$
k_{\alpha}(z, w)=\frac{1}{(1-z \bar{w})^{\alpha}} . \quad(z, w \in \mathbb{D})
$$

The purpose of this section is to extend the results of Olofsson, Ball and Bolotnikov to a large class of reproducing kernel Hilbert spaces. Our setting is very general and, as particular cases, we obtain new and simple proof of the invariant subspace theorem for vector-valued weighted Bergman spaces of Ball and Bolotnikov.

Let $K: \mathbb{D} \times \mathbb{D} \rightarrow \mathbb{C}$ be a positive definite function which is holomorphic in the first variable, and anti-holomorphic in the second variable. We denote by $\mathcal{H}_{K}$ the reproducing kernel Hilbert space corresponding to the kernel $K$.

DeFinition 3.1. Let $\mathcal{H}_{K}$ be a reproducing kernel Hilbert space with $K$ as above. We say that $\mathcal{H}_{K}$ is an analytic Hilbert space if $M_{z}$ on $\mathcal{H}_{K}$, defined by $M_{z} f=z f$ for all $f \in \mathcal{H}_{K}$, is a C.0-contraction.

Let $\mathcal{H}_{K_{1}}$ and $\mathcal{H}_{K_{2}}$ be two analytic Hilbert spaces and $\mathcal{E}_{1}$ and $\mathcal{E}_{2}$ be two Hilbert spaces. A map $\Theta: \mathbb{D} \rightarrow \mathcal{B}\left(\mathcal{E}_{1}, \mathcal{E}_{2}\right)$ is said to be a multiplier from $\mathcal{H}_{K_{1}} \otimes \mathcal{E}_{1}$ to $\mathcal{H}_{K_{2}} \otimes \mathcal{E}_{2}$ if

$$
\Theta f \in \mathcal{H}_{K_{2}} \otimes \mathcal{E}_{2} . \quad\left(f \in \mathcal{H}_{K_{1}} \otimes \mathcal{E}_{1}\right)
$$

We denote the set of all multipliers from $\mathcal{H}_{K_{1}} \otimes \mathcal{E}_{1}$ to $\mathcal{H}_{K_{2}} \otimes \mathcal{E}_{2}$ by $\mathcal{M}\left(\mathcal{H}_{K_{1}} \otimes \mathcal{E}_{1}, \mathcal{H}_{K_{2}} \otimes \mathcal{E}_{2}\right)$.

The following lemma, on a characterization of intertwining operators between a vectorvalued Hardy space and an analytic Hilbert space, is well-known, which we prove for the sake of completeness.

We will denote by $\mathbb{S}$ the Szego kernel on $\mathbb{D}$, that is,

$$
\mathbb{S}(z, w)=\frac{1}{(1-z \bar{w})} . \quad(z, w \in \mathbb{D})
$$

LEMMA 3.2. Let $\mathcal{E}_{1}$ and $\mathcal{E}_{2}$ be two Hilbert spaces and $\mathcal{H}_{K}$ be an analytic Hilbert space. Let $X \in \mathcal{B}\left(H^{2}(\mathbb{D}) \otimes \mathcal{E}_{1}, \mathcal{H}_{K} \otimes \mathcal{E}_{2}\right)$. Then

$$
X\left(M_{z} \otimes I_{\mathcal{E}_{1}}\right)=\left(M_{z} \otimes I_{\mathcal{E}_{2}}\right) X,
$$

if and only if $X=M_{\Theta}$ for some $\Theta \in \mathcal{M}\left(H^{2}(\mathbb{D}) \otimes \mathcal{E}_{1}, \mathcal{H}_{K} \otimes \mathcal{E}_{2}\right)$. 
Proof. Let $X \in \mathcal{B}\left(H^{2}(\mathbb{D}) \otimes \mathcal{E}_{1}, \mathcal{H}_{K} \otimes \mathcal{E}_{2}\right)$ and $X\left(M_{z} \otimes I_{\mathcal{E}_{1}}\right)=\left(M_{z} \otimes I_{\mathcal{E}_{2}}\right) X$. If $\zeta \in \mathcal{E}_{2}$ and $w \in \mathbb{D}$ then

$$
\left(M_{z} \otimes I_{\mathcal{E}_{1}}\right)^{*}\left[X^{*}(K(\cdot, w) \otimes \zeta)\right]=X^{*}\left(M_{z} \otimes I_{\mathcal{E}_{2}}\right)^{*}(K(\cdot, w) \otimes \zeta)=\bar{w}\left[X^{*}(K(\cdot, w) \otimes \zeta)\right],
$$

that is,

$$
X^{*}(K(\cdot, w) \otimes \zeta) \in \operatorname{ker}\left(M_{z} \otimes I_{\mathcal{E}_{1}}-w I_{H^{2}(\mathbb{D}) \otimes \mathcal{E}_{1}}\right)^{*} .
$$

This and the fact that $\operatorname{ker}\left(M_{z}-w I_{H^{2}(\mathbb{D})}\right)^{*}=<\mathbb{S}(\cdot, w)>$ readily implies that

$$
X^{*}(K(\cdot, w) \otimes \zeta)=\mathbb{S}(\cdot, w) \otimes X(w) \zeta, \quad\left(w \in \mathbb{D}, \zeta \in \mathcal{E}_{2}\right)
$$

for some linear map $X(w): \mathcal{E}_{2} \rightarrow \mathcal{E}_{1}$. Moreover,

$$
\|X(w) \zeta\|_{\mathcal{E}_{1}}=\frac{1}{\|\mathbb{S}(\cdot, w)\|_{H^{2}(\mathbb{D})}}\left\|X^{*}(K(\cdot, w) \otimes \zeta)\right\|_{H^{2}(\mathbb{D}) \otimes \mathcal{E}_{1}} \leq \frac{\|K(\cdot, w)\|_{\mathcal{H}_{K}}}{\|\mathbb{S}(\cdot, w)\|_{H^{2}(\mathbb{D})}}\|X\| \zeta \|_{\mathcal{E}_{2}},
$$

for all $w \in \mathbb{D}$ and $\zeta \in \mathcal{E}_{2}$. Therefore $X(w)$ is bounded and $\Theta(w):=X(w)^{*} \in \mathcal{B}\left(\mathcal{E}_{1}, \mathcal{E}_{2}\right)$ for each $w \in \mathbb{D}$. Thus

$$
X^{*}(K(\cdot, w) \otimes \zeta)=\mathbb{S}(\cdot, w) \otimes \Theta(w)^{*} \zeta . \quad\left(w \in \mathbb{D}, \zeta \in \mathcal{E}_{2}\right)
$$

In order to prove that $\Theta(w)$ is holomorphic we compute

$$
\begin{aligned}
\langle\Theta(w) \eta, \zeta\rangle_{\mathcal{E}_{2}} & =\left\langle\eta, \Theta(w)^{*} \zeta\right\rangle_{\mathcal{E}_{1}}=\left\langle\mathbb{S}(\cdot, 0) \otimes \eta, \mathbb{S}(\cdot, w) \otimes \Theta(w)^{*} \zeta\right\rangle_{H^{2}(\mathbb{D}) \otimes \mathcal{E}_{1}} \\
& =\langle X(\mathbb{S}(\cdot, 0) \otimes \eta), K(\cdot, w) \otimes \zeta\rangle_{\mathcal{H}_{K} \otimes \mathcal{E}_{2} .} . \quad\left(\eta \in \mathcal{E}_{1}, \zeta \in \mathcal{E}_{2}\right)
\end{aligned}
$$

Since $w \mapsto K(\cdot, w)$ is anti-holomorphic, we conclude that $w \mapsto \Theta(w)$ is holomorphic. Hence $\Theta \in \mathcal{M}\left(H^{2}(\mathbb{D}) \otimes \mathcal{E}_{1}, \mathcal{H}_{K} \otimes \mathcal{E}_{2}\right)$ and $X=M_{\Theta}$

Conversely, let $\Theta \in \mathcal{M}\left(H^{2}(\mathbb{D}) \otimes \mathcal{E}_{1}, \mathcal{H}_{K} \otimes \mathcal{E}_{2}\right)$. For $f \in H^{2}(\mathbb{D}) \otimes \mathcal{E}_{1}$ and $w \in \mathbb{D}$ this implies that

$$
(z \Theta f)(w)=w \Theta(w) f(w)=\Theta(w) w f(w)=(\Theta z f)(w) .
$$

So $M_{\Theta}$ intertwines the multiplication operators which completes the proof.

Now we are ready for the main theorem of this section.

TheOREM 3.3. Let $\mathcal{H}_{K}$ be an analytic Hilbert space and $\mathcal{E}_{*}$ be a Hilbert space. Let $\mathcal{S}$ be a non-trivial closed subspace of $\mathcal{H}_{K} \otimes \mathcal{E}_{*}$. Then $\mathcal{S}$ is $\left(M_{z} \otimes I_{\mathcal{E}_{*}}\right)$-invariant subspace of $\mathcal{H}_{K} \otimes \mathcal{E}_{*}$ if and only if there exists a Hilbert space $\mathcal{E}$ and a partially isometric multiplier $\Theta \in \mathcal{M}\left(H^{2}(\mathbb{D}) \otimes\right.$ $\left.\mathcal{E}, \mathcal{H}_{K} \otimes \mathcal{E}_{*}\right)$ such that

$$
\mathcal{S}=\Theta\left(H^{2}(\mathbb{D}) \otimes \mathcal{E}\right)
$$

Proof. By Theorem [2.2, there exists a partial isometry $\Pi: H^{2}(\mathbb{D}) \otimes \mathcal{E} \rightarrow \mathcal{H}_{K} \otimes \mathcal{E}_{*}$ such that $\Pi\left(M_{z} \otimes I_{\mathcal{E}}\right)=\left(M_{z} \otimes I_{\mathcal{E}_{*}}\right) \Pi$. Consequently, by Lemma 3.2 we have that $\Pi=M_{\Theta}$ for some $\Theta \in \mathcal{M}\left(H^{2}(\mathbb{D}) \otimes \mathcal{E}_{1}, \mathcal{H}_{K_{2}} \otimes \mathcal{E}_{2}\right)$.

The converse part is trivial. This completes the proof.

In the present context, we restate Corollary 2.3 as follows:

Corollary 3.4. Let $\mathcal{H}_{K}$ be an analytic Hilbert space and $\mathcal{S}$ be a non-trivial closed subspace of $\mathcal{H}_{K} \otimes \mathcal{E}_{*}$ for some coefficient Hilbert space $\mathcal{E}_{*}$. Then $\mathcal{S}$ is $\left(M_{z} \otimes I_{\mathcal{E}_{*}}\right)$-invariant subspace 
of $\mathcal{H}_{K} \otimes \mathcal{E}_{*}$ if and only if there exists a Hilbert space $\mathcal{E}$ and a multiplier $\Theta \in \mathcal{M}\left(H^{2}(\mathbb{D}) \otimes\right.$ $\left.\mathcal{E}, \mathcal{H}_{K} \otimes \mathcal{E}_{*}\right)$ such that

$$
P_{\mathcal{S}}=M_{\Theta} M_{\Theta}^{*}
$$

For each $\alpha>1$, the weighted Bergman space $L_{a, \alpha}^{2}(\mathbb{D})$ satisfies the conditions of Theorem 3.3. In particular, Theorem 3.3 includes the result by Ball and Bolotnikov [4] for weighted Bergman spaces with integer weights as special cases.

\section{CONCluding REMARKS}

A bounded linear operator $T$ on a Hilbert space $\mathcal{H}$ is said to have the wandering subspace property if $\mathcal{H}$ is generated by the subspace $\mathcal{W}_{T}:=\mathcal{H} \ominus T \mathcal{H}$, that is,

$$
\mathcal{H}=\left[\mathcal{W}_{T}\right]=\overline{\operatorname{span}}\left\{T^{m} \mathcal{W}_{T}: m \in \mathbb{N}\right\} .
$$

In that case we say that $\mathcal{W}_{T}$ is a wandering subspace for $T$.

An important consequence of the Beurling theorem [3] states that: given a non-trivial closed shift invariant subspace $\mathcal{S}$ of $H^{2}(\mathbb{D})$, the subspace $\mathcal{W}_{\left.M_{z}\right|_{\mathcal{S}}}=\mathcal{S} \ominus z \mathcal{S}$ is a wandering subspace for $\left.M_{z}\right|_{\mathcal{S}}$. The same conclusion holds for the Bergman space [1] and the weighted Bergman space with weight $\alpha=3$ [14] but for $\alpha>3$, the issue is more subtle (see [8], [10]). In particular, partially isometric representations of $M_{z}$-invariant subspaces of analytic Hilbert spaces seems to be a natural generalization of the Beurling theorem concerning the shift invariant subspaces of the Hardy space $H^{2}(\mathbb{D})$.

Finally, it is worth stressing that the main results of this paper are closely related to the issue of factorizations of reproducing kernels. As future work we plan to extend our approach to several variables and address issues such as factorizations of kernel functions and containment of shift-invariant subspaces of analytic Hilbert spaces over general domains in $\mathbb{C}^{n}$.

Acknowledgement: We would like to thank Joseph A. Ball and Scott McCullough for kindly and quickly answering some questions concerning reproducing kernel Hilbert spaces. We also thank Bhaskar Bagchi and Dan Timotin for numerous useful comments and suggestions on a preliminary version of this paper.

\section{REFERENCES}

[1] A. Aleman, S. Richter and C. Sundberg, Beurlings theorem for the Bergman space, Acta Math., 177 (1996), 275-310.

[2] N. Aronszajn, Theory of reproducing kernels, Trans. Amer. Math. Soc. 68 (1950), 337-404.

[3] A. Beurling, On two problems concerning linear transformations in Hilbert space, Acta Math., 81 (1949), 239-255.

[4] J. Ball and V. Bolotnikov, A Beurling type theorem in weighted Bergman spaces, C. R. Math. Acad. Sci. Paris 351 (2013), no. 11-12, 433-436.

[5] J. Ball and V. Bolotnikov, Weighted Bergman spaces: shift-invariant subspaces and input/state/output linear systems, Integral Equations Operator Theory,, 76 (2013), 301-356.

[6] R. Douglas and V. Paulsen, Hilbert Modules over Function Algebras, Research Notes in Mathematics Series, 47, Longman, Harlow, 1989.

[7] P. Halmos, Shifts on Hilbert spaces, J. Reine Angew. Math. 208 (1961), 102-112. 
[8] H. Hedenmalm and Y Perdomo, Mean value surfaces with prescribed curvature form, J. Math. Pures Appl., 83 (2004), pp. 1075-1107.

[9] P. Lax, Translation invariant spaces, Acta Math. 101 (1959) 163-178.

[10] S. McCullough and S. Richter, Bergman-type reproducing kernels, contractive divisors, and dilations, J. Funct. Anal. 190 (2002), no. 2, 447-480.

[11] B. Sz.-Nagy and C. Foias, Harmonic Analysis of Operators on Hilbert Space, North Holland, Amsterdam, 1970.

[12] A. Olofsson, A characteristic operator function for the class of n-hypercontractions, J. Funct. Anal., 236 (2006), 517-545.

[13] H. Radjavi and P. Rosenthal, Invariant subspaces, Ergebnisse der Mathematik und ihrer Grenzgebiete, Band 77, Springer-Verlag, New York-Heidelberg, 1973.

[14] S. Shimorin, Wold-type decompositions and wandering subspaces for operators close to isometries, J. Reine Angew. Math. 531 (2001), 147-189.

Indian Statistical Institute, Statistics and Mathematics Unit, 8th Mile, Mysore Road, BANGALORE, 560059, INDIA

E-mail address: jay@isibang.ac.in, jaydeb@gmail.com 